\title{
Timing of Binary Pulsars at Kalyazin, Russia
}

\author{
Yu. P. Ilyasov, V. V. Oreshko, V. A. Potapov, A. E. Rodin \\ Pushchino Radio Astronomy Observatory, P. N. Lebedev Physical \\ Institute, 142290, Pushchino, Russia
}

\begin{abstract}
Regular high-precision timing of the binary pulsars J06130200, J1012+5307, J1022+1001, J1640+2224, J1643-1224, J1713+0747, $\mathrm{J} 2145-0750$ and the pulsar B1937+21 has been conducted at the Kalyazin (Russia) radio telescope RT-64 at $0.6 \mathrm{GHz}$ over more than 6 years. Several of the pulsars monitored have been found to be good probes for gravitational wave background (GWB) tests, while others, having a shorter orbital period, can be used for establishing a dynamical binary pulsar timescale. Upper limits for the GWB energy density were estimated.
\end{abstract}

\section{Introduction}

The RT-64 radio telescope has a quasi-parabolic main reflector and tuned subreflector with a Kasegrane feed system. Diameter of the secondary mirror is $6 \mathrm{~m}$. RMS of the surface is about $1.5 \mathrm{~mm}$, and antenna noise temperature is $15-30 \mathrm{~K}$. A multi-frequency horn is used as the primary feed from 0.6 to $22 \mathrm{GHz}$ (Ilyasov, Poperechenko \& Oreshko 2000). Low-noise amplifiers are installed near the horn and cooled with helium. The filterbank used at at $0.6 \mathrm{GHz}$ has $3.2 \mathrm{MHz}$ total bandwidth $(80 \times 40 \mathrm{kHz}$ for each of two orthogonal circular polarizations). The total system temperature is about $100 \mathrm{~K}$ and defined mostly by sky background and pre-amplifiers. The data acquisition system (DAS) is synchronized by a master clock. Start time and apparent pulsar period are calculated on-line (Ilyasov, Oreshko \& Doroshenko 2000). The local time standard is based on a hydrogen maser and rubidium standards, and is compared with UTC via GPS.

\section{Observations and Results}

Pulsar timing observations are made by accumulation of pulses in the DAS RAM synchronously with the apparent pulsar period. Dispersion distortions are removed off-line. Good signal-to-noise ratio allows measurement of TOAs with up to $0.5 \mu \mathrm{s}$ accuracy. Barycentric TOA residuals are calculated using the TIMAPR (Doroshenko \& Kopeikin 1990) and TEMPO (Taylor \& Weisberg 1989) packages. Timing solutions were obtained for five binary pulsars and the isolated millisecond pulsar B1937+21. Post-fit residuals are shown in Figure 1 . Timing data for PSR J1640+2224 were used to estimate an upper limit for the relative density of GWB energy $\Omega_{g}$ (Kopeikin 1997). $\Omega_{g} h^{2}<8.5 \times 10^{-4}$ in frequencies $10^{-11}-7.1 \times 10^{-9} \mathrm{~Hz}$ ( $h$ is the dimensionless Hubble constant). 

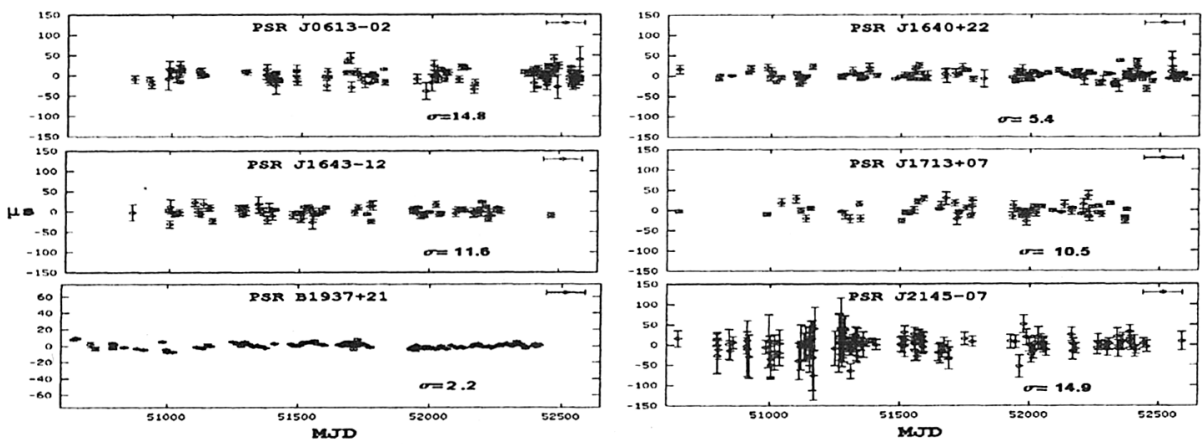

Figure 1. Post-fit residuals of millisecond and binary pulsars.

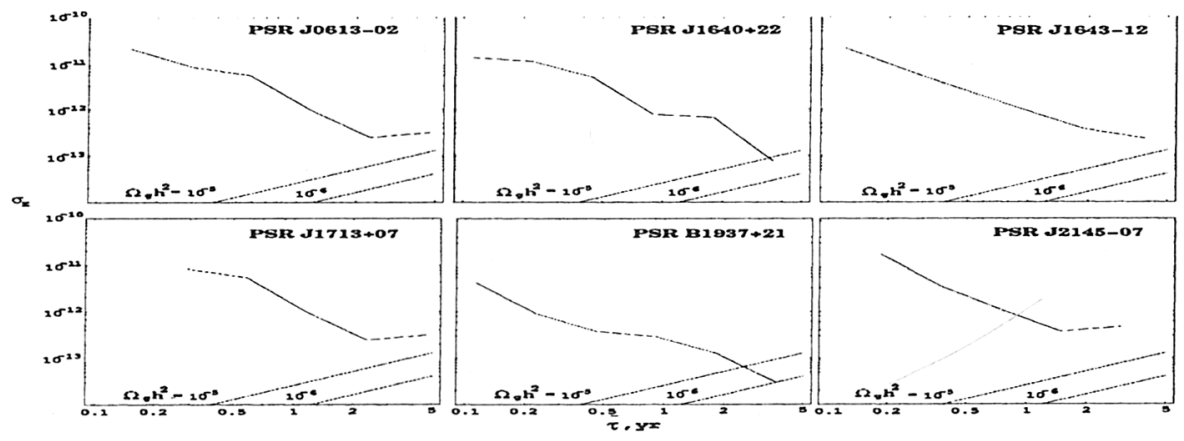

Figure 2. Fractional instabilities $\sigma_{z}\left(\Omega_{g} h^{2}\right.$ lines are shown).

Fractional instabilities $\sigma_{z}$ (Matsakis, Taylor \& Eubanks 1997) were calculated for six pulsars (see Fig. 2). Limits for GWB at a frequency of about $10^{-8}$ $\mathrm{Hz}$ are estimated as $\Omega_{g} h^{2}<10^{-6}$ for PSR B1937+21.

Regular observations of binary pulsars at Kalyazin are in progress.

Acknowledgments. The authors express gratitude to I. Lukin, A. Serov and E. Zmeeva. This work was supported by the Ministry of Industry, Science and Technologies of Russia, and RFBR grants No. 01-02-16187, 03-02-16911.

\section{References}

Doroshenko, O. V., \& Kopeikin, S. M. 1990, Sov. Astr., 34, 496

Ilyasov, Yu. P., Oreshko, V. V, \& Doroshenko, O. V. 2000, in Proc. LPI. 229, p. 95

Ilyasov, Yu. P., Poperechenko, B., \& Oreshko, V. V. 2000, in Proc. LPI. 229, p. 44

Kopeikin, S. M., 1997, Phys. Rev. D, 56, 4455

Matsakis, D. N., Taylor, J. H., \& Eubanks, T. M. 1997, A\&A, 326, 924

Taylor, J. H., \& Weisberg, J. M. 1989, ApJ, 345, 434 